beasts in their own way.

We are now familiar enough with the quantum entities. We know that wave and particle descriptions give us but partial views. For a wave is continuous and extended, a particle is discrete and localized, while quantum beings are discrete and extended. It is only in very special circumstances, for a rather restricted set of experimental devices, that the waveparticle duality suffices to give us a satisfying account. To understand most modern experiments, we must use the fullfledged apparatus of quantum theory to which the wave-particle duality has no relevance whatsoever. Even rather simple experimental situations do not lend themselves to natural explanations in terms of wave and particles, whether in alternative (either... or ...) or comprehensive (both ... and . . .) use $\mathrm{e}^{2.3}$.

It seems more than time to recognize that quantum entities are neither waves, nor particles. As the basic objects of a non-classical theory, they certainly deserve a new, non-classical, and specific name ${ }^{4}$. A natural solution is to encompass all their species, photon, electrons, baryons, gluons etc., under the common appellation of 'quantons', as long advocated by Bunge and others. This would not only dispose of the cumbersome and ill-defined 'wave-particle duality' but would also offer definite pedagogical help by stressing for the student the radical novelty of quantum theory and the danger of naive classical pictures 5 .

Finally, it would remind us that the debate about the foundations of quantum theory is not only a matter of interpretation and that there remain open questions concerning the physical theory itself ". The main problem is to understand how large pieces of matter built out of quantons can behave like waves, or like particles or like neither. In other words, rather than interpreting quantum theory, we need to understand classical theories, that is, to master the conditions of validity of classical theories? ${ }^{7}$. It is to be expected that such an understanding will pave the way to a complete solution of the most irritating riddle of quantum theory, that of the 'reduction of the state vector' under a measurement, which could be but an efficient recipe following from the macroscopic nature of the measuring apparatus.

Physique Théorique,

$$
\text { J.-M. LÉVY-LEBLOND }
$$

Université de Nice,

Parc Valrose,

06034-Nice Cedex, France

Maddox. J. Nature 332, 581 (1988)

Wootters, W.K. \& Zurck, W.H. Phys. Rev. D19, 473 (1979) Grcenberger, D.M. \& Yasin, A. Phys, Lett. A128, 391 (1988)

4. Lévy-Leblond, J.-M. Physica (in the press).

5. Lévy-Leblond, J.-M. \& Balibar. F. Quantique (Rudiments) Lévy-Lcblond, J.-M. \& Balibar, F. Quantique (Rudiments)
(Interéditions-CNRS, Paris, 1984); English translation (North-Holland. in the press)

6. Lévy-Leblond. J.-M. Int. J. Quantum Chem. XII, suppl. 1. 415-421 (1977)

7. Lévy-Leblond. J.-M. Eur. J. Phys. 2, 44-47 (1981)

\section{Cystic fibrosis allele segregation}

SIR-Kitzis et al.' presented data on normal siblings of cystic fibrosis (CF) patients, suggesting segregation distortion of the CF allele with sex. In their study, 20 of 22 normal homozygotes were girls, and 16 of 21 heterozygotes carrying the paternal CF chromosome were boys. In contrast, the maternal CF chromosome had been passed equally to boys and girls. The authors suggested that there is preferential inheritance of the $\mathrm{CF}$ allele from male to male, but this conflicts with the generally observed equal proportions of male and female CF patients. Further data were requested to determine whether their result can be confirmed.

In a sample of comparable size consisting of normal siblings of Dutch $\mathrm{CF}$ patients, we find no indication of an abnormal segregation of the CF allele: 10 of the 20 normal homozygotes are girls and 11 of 19 heterozygotes with the paternal CF chromosome and 13 of 18 heterozygotes with the maternal CF chromosome are males.

The study of Kitzis et al. was motivated by the repeatedly reported suggestion of increased fertility of $\mathrm{CF}$ carriers. About an equal number of studies, however, do not confirm this suggestion ${ }^{2}{ }^{4}$. These studies are seldom cited. It has also been shown on several occasions that sampling of families through affected children introduces a bias in favour of larger families $^{5.6}$. Methods for correction of this ascertainment bias are available ${ }^{5.6}$.

L. P. TEN Kate

G. J. TE MEERMAN C.H.C.M. BuYs

Department of Human Genetics,

University of Groningen, Groningen, The Netherlands

D.J.J. HALley

Department of Clinical Genetics,

B. OOSTRA

Erasmus University,

Rotterdam, The Netherlands

1. Kitzis, A. et al. Nature 333,215 (1988)

. Brunecký, Z., Laxová, R.\& Kamarýt, J. Pädiatrieou Grenzgeb 8, 99-107(1969)

Simankova, N. \& Vavrova, V. Cesk. Pediat. 25, 77-78 (1970).

4. Conneally, P.M. Yu. P.L. \& Merrit, A.D. Abstr. 12 th CF Club Meeting (1971).

5. Ten Kate, L.P. Ann, hum. Gener. 40, 287-297 (1977)

6. Jorde, L.B.\& Lathrop, G.M. Am. J. hum. Genet. 42, 808-813(1988).

SIR-Kitzis et al. (Nature 333, 215; 1988) presented interesting and unusual data on the segregation of the cystic fibrosis (CF) mutant allele to male and female progeny. However, the explanation and implications proffered are not consistent with their results. They found that, of the phenotypically normal progeny of (presumably) heterozygous parents, most homozygous normal progeny were females, most heterozygotes carrying the paternal CF allele were male, and that male and female heterozygotes carrying the material $\mathrm{CF}$ allele were equally represented. The authors claim that, because there is preferential inheritance from male to male, that this provides a mechanism for expansion of the CF allele in caucasian populations which exhibit unexpectedly high frequencies of this otherwise deleterious allele. Closer examination of their data shows that the ratio of mutant to normal alleles in the progeny $(38: 82)$ is close to the expected $1: 2$. There were 21 heterozygous progeny carrying the paternal $\mathrm{CF}$ allele compared to the 20 expected. There is no mechanism here which would promote CF mutants over normal alleles.

The authors go on to suggest that the $\mathrm{CF}$ allele may be linked to some factor causing differential sperm viability or embryo survival. If so, one would expect the overall frequency, but not the sex ratio, of heterozygous offspring carrying the paternal CF allele to be affected. To explain the distorted sex ratio of these heterozygotes, the factor affecting viability would have to have differential effects depending on the presence of the paternal $\mathrm{X}$ or $\mathrm{Y}$ chromosome. The difficulty would be in explaining why there is such a marked deficit of male homozygous normal progeny.

An associated segregation of the CF allele (on chromosome 7) and the $\mathrm{Y}$ chromosome would explain the observed imbalanced sex ratios. However, one would also then expect more females than males among heterozygous offspring carrying the maternal $\mathrm{CF}$ allele. The authors do not give results for this class of offspring, other than to say that equal numbers of males and females were observed (which is strictly impossible as there were 17 progeny in total). Assuming that the number of females differ from the number of males by only one, either way, fitting the observed row and column totals and the observed average association between the CF allele and the Y chromosome, gives a residual $\chi_{1}^{2}>7.63, P<$ 0.01 , thus failing adequately to explain the observations. This explanation would also require that most $\mathrm{CF}$ homozygotes were male, which is known not to be the case.

The conclusion must be that the results of Kitzis et al. are puzzling and not explained by any simple mechanism. Nor do they explain the surprisingly high frequency of the CF allele. Further speculation on the matter should probably be left until these results are confirmed, or otherwise.

\section{Centre for Genetic Improvement of}

Livestock,

Department of Animal and Poultry

Science,

University of Guelph,

Ontario, Canada N1G $2 W 1$ 